\title{
Evolution of Discrete Opinions on Human-Swarm Interaction in Influence Networks
}

\author{
Feres Azevedo Salem* Renan da Silva Tchilian* \\ Ubirajara Franco Moreno* \\ * Federal University of Santa Catarina, Automation and Systems \\ Department, Florianopolis, Brazil, (e-mails: \\ feres.salem@posgrad.ufsc.br, renan.tchilian@posgrad.ufsc.br, \\ ubirajara.f.moreno@ufsc.br).
}

\begin{abstract}
The aim of this paper is to explore social dynamics in conjunction with robot swarms dynamics to reach opinion consensus in a discussions series. First, it is presented the DeGroot-Friedkin model for the self-confidence dynamics of individuals and how it is updated to discussions on a sequence of topics, reaching a consensus in each case. Also, it is proposed an adaptation to a gossip-based algorithm in order to handle the consensus of opinions limited to a finite set of possible values. After each issue discussion, the interactions weights are updated. Then it is considered the case where some agents are replaced by robotic agents and it is analyzed how this impact the discussion outcome compared to the previous case. It is concluded that these agents with static self-confidence make others less confident and consequently have a greater influence on the discussion over a sequence of topics outputs.
\end{abstract}

Keywords: opinion dynamics, consensus algorithms, human-swarm interactions, social network, multi-agent systems.

\section{INTRODUCTION}

The literature on multi-robots systems brings applications to consensus dealing with continuous opinions, i.e., where the opinion of a robot on an issue belongs to the set of real numbers. As examples, problems of formation control (Correia and Moreno, 2015), where the robots need to synchronize its velocities, and rendezvous in space (Salem et al., 2018), where the robotic team decides the best point to meet.

However, in other situations, the agents may be asked to decide between a finite set of possible choices. This kind of decision will be further referenced in this paper as consensus with discrete opinions.

There are many examples of those decisions processes among groups of humans. Both on small scale, such as deciding a pizza flavor or which movie to watch on theaters, and large scale, when voting for the approval of a law bill or choosing the best candidate in an election.

Groups of human agents are commonly called in the literature as social or influence networks. There are plenty of fields and techniques that are concerned about this matter, such as psychology analysis (Smith and Collins, 2009), Markov chains (Bolzern et al., 2019), stochastic models (Wang et al., 2018), evolutionary game theory (Yu et al., 2016), coevolutionary dynamics (Jia et al., 2016), collective intelligence (Sulis, 2009) and reinforcement learning (Zhang et al., 2018).

\footnotetext{
^ This work was supported by CAPES, Brazil.
}

The systems and control community studies better the dynamics of real-valued opinions since it is described by differential or recurrent equations (Proskurnikov and Tempo, 2018). Although some models that deals with quantized communication (that is, the information exchanged by the agents is limited to a finite set of symbols) can be found at the works of Ceragioli and Frasca (2018); Martins (2013); Urbig (2003), it was decided to modify a model originally proposed to the dynamics of continuous opinions.

The model chosen was the DeGroot-Friedkin for opinion dynamics that includes the evolution of social power based on self-appraisals from the individual (Jia et al., 2015). The main reason for this choice is because this model addresses the evolution of the influence weights across a sequence of issues being discussed by the group.

The contribution of this paper is providing the mathematical description of the relative interactions matrix, from the DeGroot-Friedkin model, for a scenario where some of the nodes are robots. Furthermore, is proposed an opinion dynamics model for handling discrete opinions which benefits from the influence weights that the DeGroot-Friedkin model outputs.

Also, some simulations results are presented to illustrate the behavior of the model in two meaningful scenarios followed by some analysis of the opinion consensus, the number of iterations and comparisons between initial selfweights and stabilized self-weights. Moreover, the results with a larger group are shown to demonstrate the model scalability. 
The rest of the paper is organized as follows. Section 2 features the DeGroot-Friedkin model and the inclusion of agents with static weights. Section 3 presents an asynchronous asymmetric gossip-based model to define the opinion dynamics. Section 4 presents the analysis results for the two meaningful scenarios, in which the first one the relative interpersonal influences dynamics obey the DeGroot-Friedkin model i.e. the system is homogeneous (only human agents) and the second scenario in which the system is heterogeneous (human and robot agents). Section 5 contains our conclusions and further works.

\section{THE DEGROOT-FRIEDKIN MODEL}

The DeGroot-Friedkin model presented by Jia et al. (2015) predicts the evolution of an influence network governing the process of opinion formation based on the relative interpersonal weights. The model predicts that the social power ranking among individuals evolves across issues and asymptotically tends to their centrality score. These analyses of issue sequences and this proposed formalization of evolution were motivated by the sociological hypothesis of reflected appraisals by Cooley (2017).

The available empirical evidence is also consistent with the assumption that individuals update their opinions as convex combinations of their own and the displayed opinions by others, based on weights that are automatically generated by individuals in their responses to the displayed opinions of others (Jia et al., 2015).

Based upon this, a social influence network can be described as a weight matrix $W=\left[w_{i j}\right]$ satisfying $w_{i j} \in[0,1]$ for all $i$ and $j$ and $\sum_{j} w_{i j}=1$ for all $i$ (that is, $W$ is rowstochastic). Each edge of this network $j \stackrel{w_{i j}}{\longrightarrow} i$, including self-loops $i \stackrel{w_{i i}}{\longrightarrow} i$,represents the influence weight accorded by agent $i$ to agent $j$. Representing the opinions of the individuals with a real-valued vector $y$, the classic DeGroot model (DeGroot, 1974) is given by (1).

$$
y(k+1)=W y(k), \quad k=1,2, \ldots
$$

Sustained on empirical observations, Jia et al. (2015) combines the DeGroot model (1) of opinion dynamics, in which the influence network for a particular issue is fixed, with Friedkin (2011) formalization to the evolution of interpersonal influences in an issue sequence. The resulting dynamical process is referred as the DeGroot-Friedkin model and is given as follows

$$
y(s, k+1)=W(s) y(s, k), \quad s=1,2, \ldots, k=1,2, \ldots
$$

where $s$ indicates the issue that is being discussed and $W(s)=\left[w_{i j}(s)\right]$ is the issue-dependent matrix of influence weights.

From (2) it is possible to notice that in the DegrootFriedkin model it is assumed that timescales for the two processes are separate: the opinion dynamics are faster than the reflected appraisal dynamics in the influence network. In other words, opinion consensus is achieved before individual self-weights are updated.
For notation simplicity, the self-weights, $w_{i i}(s)$, will be denoted as $x_{i}(s)$ and they are the core of the model, since the interpersonal weights are evaluated using their values. Each weight $w_{i j}(s), i \neq j$, satisfies $w_{i j}(s)=\left(1-x_{i}(s)\right) c_{i j}$, where the relative interpersonal weights $c_{i j}$ are static and issue independent.

In an influence network with $n \geq 2$ agents, the self-weights are updated as $x(s+1)=F(x(s))$, where $F: \Delta_{n} \rightarrow \Delta_{n}$ ( $\Delta_{n}$ being the $n$-simplex) is a continuous map defined by

$F(x)= \begin{cases}\mathbb{E}_{i} & , x=\mathbb{e}_{i} \forall i \\ \left(\frac{\sigma_{1}}{1-x_{1}}, \ldots, \frac{\sigma_{n}}{1-x_{n}}\right)^{T} / \sum_{i=1}^{n} \frac{\sigma_{i}}{1-x_{i}}, & \text { otherwise }\end{cases}$

where $\mathbb{e}_{i}$ is the $i$ th basis vector (all elements equal to 0 except for the $i$ th that is equal to 1$), \sigma^{\top}=\left[\sigma_{1} \ldots \sigma_{n}\right]$ is the vector of centrality scores for each agent and it is defined as the dominant left eigenvector of the relative interaction matrix $C=\left[c_{i j}\right] \in \mathbb{R}^{n \times n}$ that is row-stochastic, zero-diagonal, and irreducible.

\subsection{Including agents with static self-weights}

Considering now the DeGroot-Friedkin model with two types of agents. The first one are the human-agents whose dynamics of interpersonal weights are described by equation (3). And the second type are the robot-agents whose self-weights are static as well as their interpersonal weights. Therefore the relative interaction matrix $C$ can be divided in four components:

$$
C=\left[\begin{array}{ll}
C_{n_{h} \times n_{h}}^{h h} & C_{n_{h} \times n_{r}}^{r h} \\
C_{n_{r} \times n_{h}}^{h r} & C_{n_{r} \times n_{r}}^{r r}
\end{array}\right]
$$

The meaning of each subscript and superscript of the relative interaction matrix $C$ is given below:

- $n_{h}$ : number of human-agents.

- $n_{r}$ : number of robot-agents.

- $h h$ : interpersonal relations human $\rightarrow$ human.

- $h r$ : interpersonal relations human $\rightarrow$ robot.

- $r h$ : interpersonal relations robot $\rightarrow$ human

- $r r$ : interpersonal relations robot $\rightarrow$ robot.

Thus the total number of agents is given by:

$$
n=n_{h}+n_{r}
$$

The submatrices of $C$ have their elements defined as

$$
C^{h h}=\left[c_{i j}^{h h}\right] ; C^{h r}=\left[c_{i j}^{h r}\right] ; C^{r h}=\left[c_{i j}^{r h}\right] ; C^{r r}=\left[c_{i j}^{r r}\right]
$$

where the indices $i$ and $j$ denotes the absolute position of the element in the whole matrix $C$. Since matrix $C$ is rowstochastic with zero diagonal, we have that the relative interpersonal weights must obey the following constraints:

$$
c_{i i}^{h h}=0 ; c_{i i}^{r r}=0
$$




$$
\begin{gathered}
\sum_{j=1}^{n_{h}} c_{i j}^{h h}+\sum_{j=n_{h}+1}^{n} c_{i j}^{r h}=1 \\
\sum_{j=1}^{n_{h}} c_{i j}^{h r}+\sum_{j=n_{h}+1}^{n} c_{i j}^{r r}=1 \\
\sum_{j=1}^{n_{h}} c_{i j}^{h r} \triangleq \frac{1}{n-1} \\
\sum_{j=n_{h}+1}^{n} c_{i j}^{r r} \triangleq \frac{n-2}{n-1}
\end{gathered}
$$

Hence, with the insertion of agents with different weights dynamics than the stipulated by equation (3) it is desired to alter the output of a opinion formation process that consider a finite set of possible opinions.

\section{GOSSIP-BASED CONSENSUS}

It is unrealistic to assume that groups of humans will act synchronously when arguing about a given issue. Instead of agents simultaneously updating their opinions, it is possible to assume that they interact in pairs. This approach is known as gossiping, which consists of two agents interacting at any step and, after that, one or both of their opinions can be changed (Proskurnikov and Tempo, 2018).

For this paper, it will be considered an asynchronous asymmetric gossip model. It is based on a model shown in the work of Fagnani and Zampieri (2007), which consider a group of $n$ agents with opinions $y_{1}(k), \ldots, y_{n}(k) \in \mathbb{R}$ and a stochastic matrix $P=\left[p_{i j}\right]$ with $p_{i i}=0$.

At each step $k$ of the opinion iteration loop, one agent $i=i(k)$ is randomly activated; the sequence $i(k)$ is independent and identically distributed and uniformly distributed in $1, \ldots, n$. The active agent $i$ has probability $p_{i j}$ of interacting with agent $j$. Whenever an interaction occurs, the opinion of agent $i$ is updated as follows

$$
y_{i}(k+1)=\left(1-\gamma_{i}\right) y_{i}(k)+\gamma_{i} y_{j}(k)
$$

where $\gamma_{i} \in(0,1)$ is a constant, describing the "trust" of agent $i$ in its neighbors. The opinions of the other agents (including $j$ ) remains unchanged

$$
y_{l}(k+1)=y_{l}(k) \quad \forall l \neq i(k)
$$

The model shown in (11) calculates a weighted average between the opinions of agents $i$ and $j$, which is not applicable when dealing with a finite set of possible opinions. In order to achieve consensus under that assumption, some considerations are presented in the following section.

\subsection{Handling discrete opinions}

The first adaptation to the model in (11), was defining that $\gamma_{i}=1, \forall i$. This implicates that whenever a pair $(i, j)$ is randomly selected, agent $i$ will replace its opinion entirely by the one from agent $j$. Therefore, at each time step, the active agent $i(k)$ updates its opinion, with probability $p_{i j}$, as follows

$$
y_{i}(k+1)=y_{j}(k)
$$

while the inactive agents remains with their opinion unchanged, as described in (12).

This modification guarantees that the final consensus opinion will belong to the set of initial opinions, being that finite set the only possibles opinion states that the agents can assume.

\subsection{The role of the influence weights}

To connect the proposed model of consensus over discrete opinions with the applications in social and influence networks, another assumption must be made. It is not sensible to think that humans will trade their opinions for another completely at random. So, a more realistic assumption is to consider that the probability of agent $i$ to replace its opinion by the opinion of agent $j$ will depend on the influence that $j$ has over $i$.

Finally, the mathematical way to make this assumption will be to consider the matrix of interactions probabilities, $P$, from the gossip-based consensus equal to the matrix of influence weights, $W$, from the DeGroot-Friedkin model. That means

$$
P \triangleq W(s)
$$

\section{COMPUTATIONAL EXPERIMENTS}

Once presented the DeGroot-Friedkin model for the opinion dynamics and the evolution of social power in influence networks, followed by the modifications to consider human-swarm interactions and the asynchronous asymmetric gossip model adapted for communication of the opinions' finite set, it is appropriate to conduct analysis for the conjunction proposed. In this section, it is presented the algorithm developed to conduct the numerical simulations; descriptions of the proposed scenarios and at last the simulation results obtained for the scenarios considered.

\subsection{The algorithm}

Algorithm 1 represents the pseudocode to carry the simulations, separated into three stages: parameter settings (beginning), issues iterations (outer loop) and consensus iterations (inner loop). It is relevant to notice that the selfweights dynamics influences the opinion consensus, but the contrary is not true.

\subsection{Proposed scenarios}

It is presented here two meaningful scenarios. The first scenario consists of ten agents with weight-dynamics according to the DeGroot-Friedkin model and it is chosen an asynchronous asymmetric gossip model to the discussion of issues. In the second scenario, it is maintained the gossip model, however, it is assumed two types of agents, eight as in the first scenario and two with static self-weights.

Then it is analyzed the opinion consensus, the number of iterations and the self-weights evolution. Furthermore, it is considered if the fact of some agents self-confidence being 


\section{Algorithm 1. Steps of the computational experiment}

Set the parameters of the experiment:

define number of humans, $n_{h}$, number of robots, $n_{r}$, and the initial self-weights of all nodes, $x(1)=$ $\left[x_{1}(1), \ldots, x_{n_{h}}(1), \ldots, x_{n}(1)\right]$, where $n=n_{h}+n_{r}$

create a random row-stochastic matrix $C=\left[c_{i j}\right]$ that respect the specifications given in (6-10)

compute the eigenvector centrality scores, $\mathcal{C} \in \mathbb{R}^{n}$, for the matrix $C$ as described previously

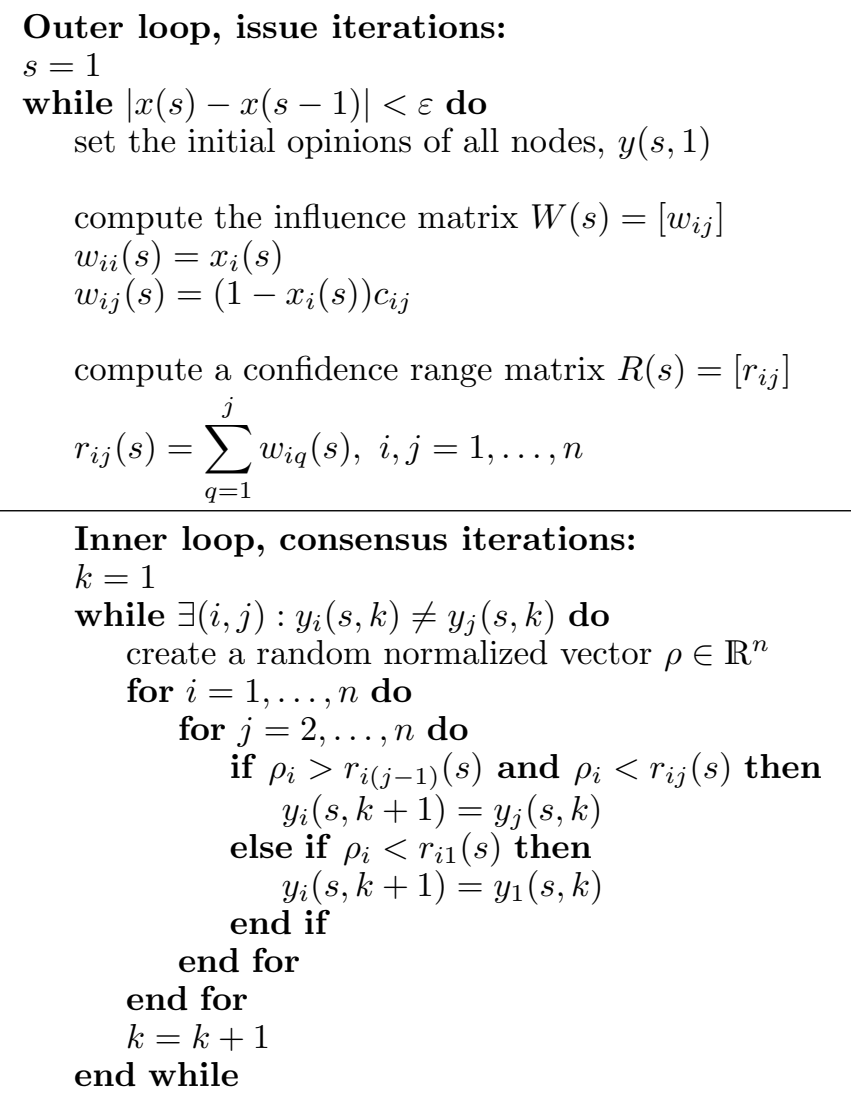

the human nodes update its self-weights by the DeGroot-Friedkin model (3)

the robotic nodes persist its self-weights across issues $x_{i}(s+1)=x_{i}(s), i=n_{h}+1, \ldots, n$

$s=s+1$

end while

stationary can affect the output of discussed issues. Lastly, the results with a larger group are shown to demonstrate the model scalability.

\subsection{Results}

The consensus outputs are observed on Table 1 for the two proposed scenarios described previously. The letters in bold for the initial opinions indicates which agents were replaced for robotic agents in the corresponding scenario. As can be verified the presence of robot agents changed the consensus information in every case, except the last one. Another interesting trait that can be observed is that, in all issues, when the robots are considered the consensus output was the initial opinion from one of them.

Table 1. Consensus outputs

\begin{tabular}{cccc}
$\begin{array}{c}\text { Issue } \\
{[s]}\end{array}$ & $\begin{array}{c}\text { Initial Opinions } \\
{[y(s, 1)]}\end{array}$ & $\begin{array}{c}\text { Final } \\
\text { without } \\
\text { robots }\end{array}$ & $\begin{array}{c}\text { Final } \\
\text { with } \\
\text { robots }\end{array}$ \\
\hline 1 & $\{\mathrm{~B}, \mathrm{~A}, \mathrm{~A}, \mathrm{C}, \mathrm{A}, \mathrm{C}, \mathrm{B}, \mathrm{C}, \mathbf{A}, \mathbf{B}\}$ & $\mathrm{C}$ & $\mathrm{A}$ \\
\hline 2 & $\{\mathrm{~B}, \mathrm{C}, \mathrm{C}, \mathrm{B}, \mathrm{B}, \mathrm{B}, \mathrm{A}, \mathrm{B}, \mathbf{A}, \mathbf{C}\}$ & $\mathrm{B}$ & $\mathrm{C}$ \\
\hline 3 & $\{\mathrm{~A}, \mathrm{C}, \mathrm{A}, \mathrm{A}, \mathrm{B}, \mathrm{A}, \mathrm{C}, \mathrm{B}, \mathbf{A}, \mathbf{A}\}$ & $\mathrm{C}$ & $\mathrm{A}$ \\
\hline 4 & $\{\mathrm{~B}, \mathrm{~B}, \mathrm{C}, \mathrm{B}, \mathrm{A}, \mathrm{C}, \mathrm{A}, \mathrm{B}, \mathbf{B}, \mathbf{C}\}$ & $\mathrm{C}$ & $\mathrm{B}$ \\
\hline 5 & $\{\mathrm{~A}, \mathrm{~A}, \mathrm{~B}, \mathrm{~B}, \mathrm{~A}, \mathrm{~B}, \mathrm{C}, \mathrm{C}, \mathbf{C}, \mathrm{B}\}$ & $\mathrm{B}$ & $\mathrm{C}$ \\
\hline 6 & $\{\mathrm{~B}, \mathrm{C}, \mathrm{C}, \mathrm{A}, \mathrm{A}, \mathrm{A}, \mathrm{A}, \mathrm{C}, \mathbf{A}, \mathbf{C}\}$ & $\mathrm{A}$ & $\mathrm{A}$ \\
\hline
\end{tabular}

Thus, in this case, only changing the dynamics of two agents in the system demonstrated that it is possible to alter the consensus opinion, even when that information is not prevalent in the beginning. Then it is observed in Figure 1 that from issue six onward the self-weights stabilize each one at a different state. Hence an equilibrium is reached, but with distinct values for each agent. Although the robot agents have static self-weights, it is important to emphasize that their opinion also changes accordingly with the gossip model, as occurs for the others.

The case when all agents' self-weights converge to the same value $(1 / n)$ is called by Jia et al. (2015) of democratic configuration and it requires the relative interaction matrix $C$ being doubly stochastic.

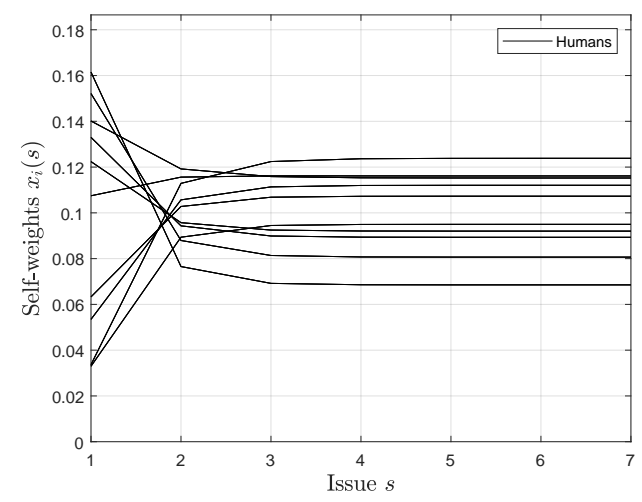

Figure 1. Self-weights evolution with 10 agents (humans).

In the second scenario, Figure 2, it is observed that from issue four onward the self-weights stabilize each one at a different state, but this time all human agents have a considerable lower self-weight when compared to the first scenario. In other words, the presence of agents with static self-confidence resulted in the other agents selfdepreciation.

The connections of all agents and their respective selfweights evolution between issues can be seen in Figure 3 in which the edge width indicates the interpersonal weight while the node width represents the self-weight value. In the first graph is presented the initial conditions and in the second graph is observed the states in which the dynamics self-weights have already stabilized.

Following the opinions evolution it is observed in Figures 4 and 5 how many nodes share the same opinion and 


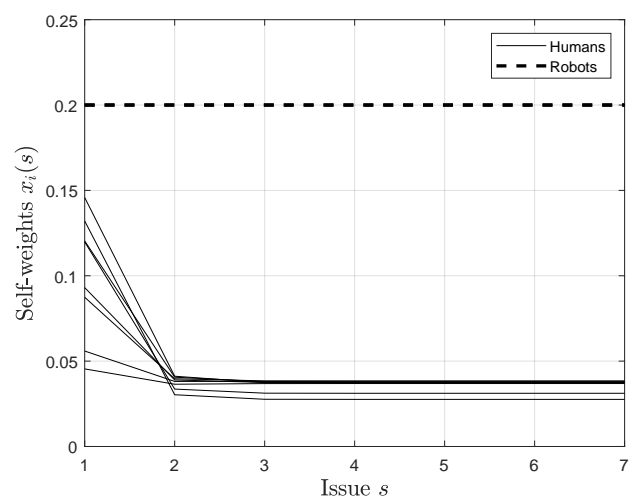

Figure 2. Self-weights evolution with 8 human agents and 2 robot agents.

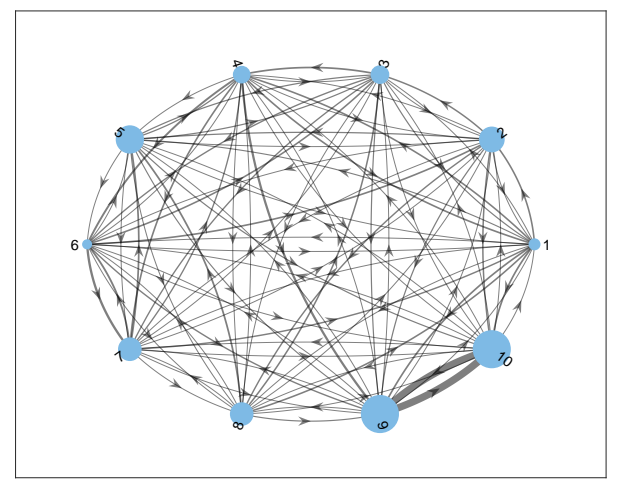

(a)

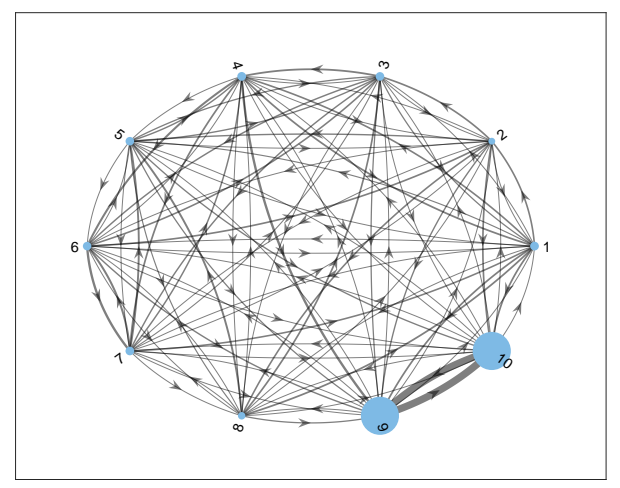

(b)

Figure 3. Evolution of the influence network across the sequence of issues: (a) initial conditions at $s=1$; (b) static state conditions at $s=4$

how many steps took to reach consensus for the initial self-weights and after their stabilization, respectively. It is important to emphasize that the two dynamics (consensus and self-weights) do not interfere in each other and it is possible to analyze them separately.

It can be seen that, for the weight's initial conditions, it was almost twice faster than when compared with the number of steps necessary to reach consensus when the self-weights have already stabilized. One hypothesis for justifying this phenomena is that the human agents being less self-confident their opinion change more easily, what may delay the consensus.

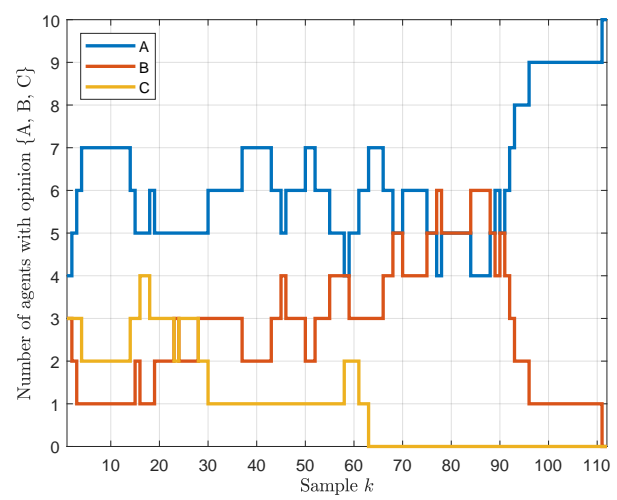

Figure 4. Opinion evolution with 8 human agents and 2 robot agents for initial self-weights $(s=1)$.

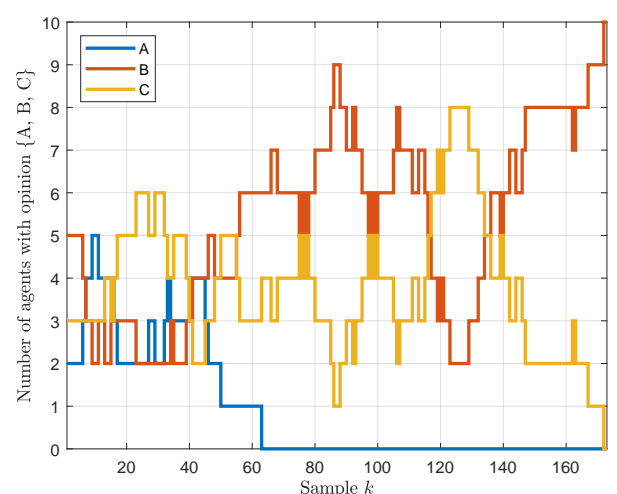

Figure 5. Opinion evolution with 8 human agents and 2 robot agents for stabilized self-weights $(s=4)$.

Finally to demonstrate the proposed analyses scalability, it is shown two scenarios with one hundred agents being 90 human agents and 10 robot agents. The first issue discussion is showed in Figure 6 and the last issue discussion are then presented in Figure 7.

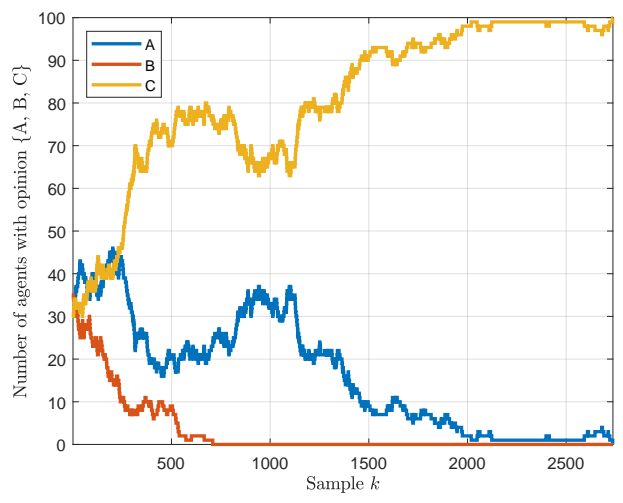

Figure 6. Opinion evolution with 90 human agents and 10 robot agents for initial self-weights $(s=1)$.

When compared to the first two simulations, this third case reaches consensus much more slower, for both the initial conditions and the final ones. This is in agreement to the notion that small groups will come to a consensus faster than bigger groups. However, the main feature here is that even with one hundred agents the system reached an opinion agreement, what means that the model is scalable 


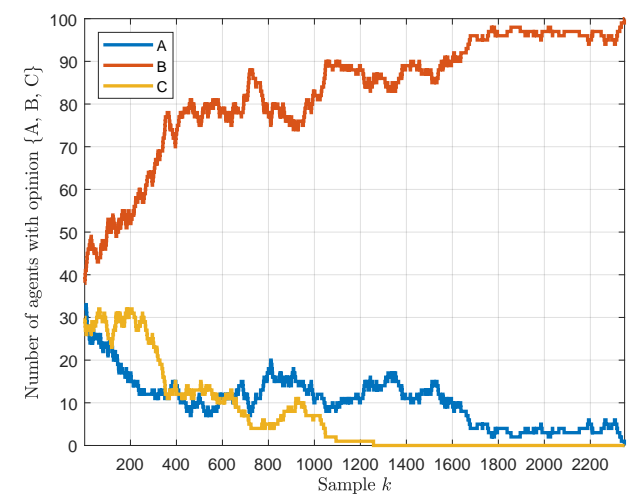

Figure 7. Opinion evolution with 90 human agents and 10 robot agents for stabilized self-weights $(s=3)$.

to handling swarms of robots interacting with huge social networks.

\section{CONCLUSIONS AND FURTHER WORKS}

The analyses presented here demonstrated the possibility to change the consensus opinion by just influencing indirectly the self-confidence of the agents. Once verified this, there are many open questions to be explored in future works. As examples, it is possible to investigate on how to define the best dynamics for the robotic agents and if its possible, by manipulating these dynamics, to guarantee the convergence for a desired consensus information.

Also, it may be studied the possibility of delaying or accelerating the consensus by manipulation of the interaction weights given from robotic agents to the humans opinions. Furthermore, it could be explored the influence of robotic agents placement when using different types of topology. Additionally to all these propositions, the DeGroot-Friedkin self-appraisal models extensions could be prospected.

One final and interesting proposal for further works is to develop a real-world experiment, where a group of volunteers would have to discuss a few issues through some interface and come to an agreement, without knowing that there are also robotic agents participating in the debate. Despite the great ambition of this experiment, it could bring key results for understanding if and how contemporary society can be misguided and, hopefully, give some insights on how to avoid it.

\section{ACKNOWLEDGMENTS}

Thanks for the resources provided by the Federal University of Santa Catarina (UFSC) and the Coordination for the Improvement of Higher Education Personnel (CAPES).

\section{REFERENCES}

Bolzern, P., Colaneri, P., and De Nicolao, G. (2019). Opinion influence and evolution in social networks: A Markovian agents model. Automatica, 100, 219-230.

Ceragioli, F. and Frasca, P. (2018). Consensus and disagreement: The role of quantized behaviors in opinion dynamics. SIAM Journal on Control and Optimization, 56(2), 1058-1080.

Cooley, C.H. (2017). Human nature and the social order. Routledge.

Correia, F.L.d.B. and Moreno, U.F. (2015). Decentralized formation tracking for groups of mobile robots with consensus and mpc. IFAC-PapersOnLine, 48(19), 274279.

DeGroot, M.H. (1974). Reaching a consensus. Journal of the American Statistical Association, 69(345), 118-121.

Fagnani, F. and Zampieri, S. (2007). Randomized consensus algorithms over large scale networks. 2007 Information Theory and Applications Workshop, Conference Proceedings, ITA, 26(4), 150-159.

Friedkin, N.E. (2011). A formal theory of reflected appraisals in the evolution of power. Administrative Science Quarterly, 56(4), 501-529.

Jia, P., Friedkin, N.E., and Bullo, F. (2016). The Coevolution of Appraisal and Influence Networks Leads to Structural Balance. IEEE Transactions on Network Science and Engineering, 3(4), 286-298.

Jia, P., MirTabatabaei, A., Friedkin, N.E., and Bullo, F. (2015). Opinion dynamics and the evolution of social power in influence networks. SIAM review, 57(3), 367397.

Martins, A.C. (2013). Trust in the coda model: Opinion dynamics and the reliability of other agents. Physics Letters A, 377(37), 2333-2339.

Proskurnikov, A.V. and Tempo, R. (2018). A tutorial on modeling and analysis of dynamic social networks. part ii. Annual Reviews in Control, 45, 166-190.

Salem, F.A., Moreno, U.F., and Castelan, E.B. (2018). Information distributed kalman filter applied to rendezvous problems in cooperative robotic teams. IFACPapersOnLine, 51(25), 190-195.

Smith, E.R. and Collins, E.C. (2009). Contextualizing Person Perception: Distributed Social Cognition. Psychological Review, 116(2), 343-364.

Sulis, W. (2009). Collective intelligence: Observations and models. In S.J. Guastello, M. Koopmans, and D. Pincus (eds.), Chaos and Complexity in Psychology: The Theory of Nonlinear Dynamical Systems, 41-66. Cambridge University Press.

Urbig, D. (2003). Attitude dynamics with limited verbalisation capabilities. Journal of artificial societies and social simulation/University of Surrey. Department of Sociology.-Guildford, 6(1), 1-23.

Wang, X., Liang, H., Fei, M., Su, H., Wang, Y., and Peng, C. (2018). Continuous-time opinion dynamics with stochastic multiplicative noises. IEEE Transactions on Circuits and Systems II: Express Briefs, $\mathrm{PP}(\mathrm{c}), 1-1$.

Yu, C., Tan, G., Lv, H., Wang, Z., Meng, J., Hao, J., and Ren, F. (2016). Modelling Adaptive Learning Behaviours for Consensus Formation in Human Societies. Scientific Reports, 6(May), 1-13.

Zhang, C., Li, X., Hao, J., Sen, S., Xue, W., and Feng, Z. (2018). The dynamics of opinion evolution in gossipermedia model with wols-cala learning. In Proceedings of the 17th International Conference on Autonomous Agents and MultiAgent Systems, AAMAS '18, 21592161. International Foundation for Autonomous Agents and Multiagent Systems, Richland, SC. 